\title{
FACTORES DOMICILIARIOS ASOCIADOS CON LA PRESENCIA DE HIDATIDOSIS HUMANA EN TRES COMUNIDADES RURALES DE JUNÍN, PERÚ
}

\author{
Saul J. Santivañez ${ }^{1, a}$, Cesar Naquira ${ }^{2,3, b}$, Cesar M. Gavidia4,c, Luis Tello ${ }^{1, d}$, Eddy Hernandez ${ }^{1, d}$, \\ Enrico Brunetti ${ }^{5, e}$, Malika Kachani ${ }^{6, f}$, Armando E. Gonzalez ${ }^{4, f}$, Hector H. Garcia $7,8,9$
}

\begin{abstract}
RESUMEN
Introducción. La hidatidosis, zoonosis producida por el estadio larvario de la taenia Echinococcus granulosus, es un problema de salud pública a nivel nacional, en especial en aquellas regiones dedicadas a la crianza de ganado. A la fecha, se han descrito factores, a nivel individual, asociados con la infección por E. granulosus; sin embargo, no se encontró reporte previo que explore la asociación entre características de la vivienda y la presencia de esta enfermedad en alguno de sus miembros. Objetivos. Explorar la asociación entre las características de la vivienda y la presencia de hidatidosis entre las personas que la habitan. Materiales y métodos. Se realizó un estudio transversal en viviendas de tres comunidades rurales de Junín. Se evaluó el grado de asociación entre las características de la vivienda y la presencia de hidatidosis en el hogar mediante un análisis de regresión logística múltiple (RLM). Resultados. De un total de 417 viviendas evaluadas, $56(13 \%)$ de ellas tenían al menos un caso positivo entre sus miembros; luego del análisis de RLM se observó que aquellas viviendas con más de tres miembros, localizadas en la comunidad con quintil de pobreza más bajo, que refirieron crianza de animales, y con una cobertura de evaluación mayor al $25 \%$ presentaron una mayor probabilidad de tener al menos un caso de positivo entre sus miembros. Conclusión. Las características observadas deben ser tomadas en cuenta para la determinación preliminar de subgrupos de alto riesgo, optimizando así el uso de los recursos y mejorando la eficacia de los programas de despistaje.
\end{abstract}

Palabras clave: Equinococosis; Asentamientos rurales, Domicilio; Perú (fuente: DeCS BIREME).

\section{HOUSEHOLD FACTORS ASSOCIATED WITH THE PRESENCE OF HUMAN HYDATID DISEASE IN THREE RURAL COMMUNITIES OF JUNIN, PERU}

\begin{abstract}
Introduction. Hydatid disease, a zoonosis caused by the larval stage of the parasite Echinococcus granulosus, is a public health problem at national level, especially in those regions dedicated to raising livestock. By now, there are many factors, at individual level, that have been associated to the infection by E. granulosus; nevertheless there is not any previous report that explore the association between household characteristics and the presence of the disease among household members. Objective. To explore the association between household characteristics and the presence of hydatid disease among household members. Material and methods. We performed a cross-sectional study in the households of 3 rural communities located in Junín, we evaluated the association between household characteristics and the presence of hydatid disease by multiple logistic regression (MLR). Results. From 417 evaluated households, $56(13 \%)$ of them had at least one positive case among its members. Multivariate analysis showed that households with three or more members, located in the community with the lowest quintile of poverty, that reported raising livestock, and with evaluation coverage greater than $25 \%$ were more likely to have at least one positive case among its members. Conclusion. The observed characteristics be taken into account in the preliminary definition of high-risk subgroups, optimizing the use of resources and improving the effectiveness of screening programs.
\end{abstract}

Key words: Echinococcosis; Rural settlements; Home; Peru (source: MeSH NLM).

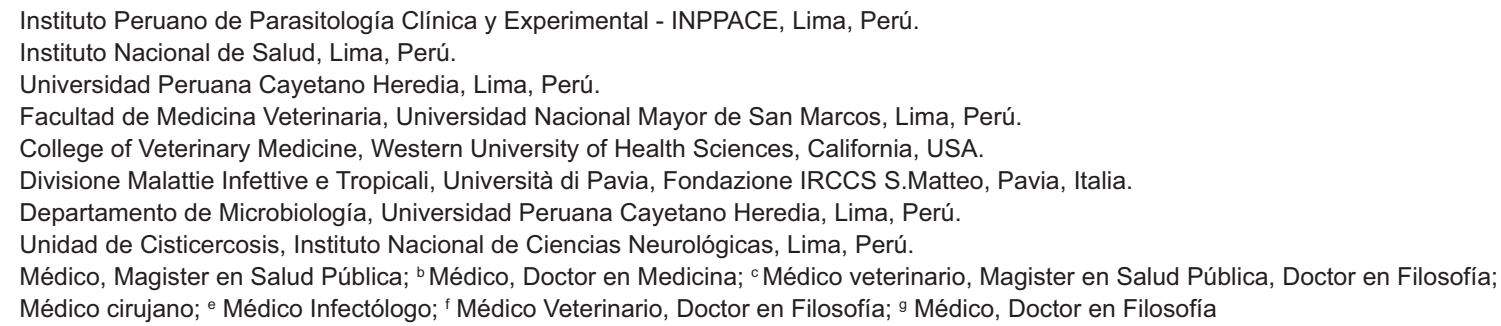




\section{INTRODUCCIÓN}

La hidatidosis es una zoonosis causada por el estadio larvario de la taenia Echinococcus granulosus. Dentro del ciclo de vida del E. granulosus, los huevos del parásito adulto son eliminados a través de las heces del perro infectado, para luego ser ingeridos por un huésped intermediario (ovejas, vacas, cabras, cerdo, o caballos, entre otros mamíferos). Una vez ingeridos, los huevos eclosionan a nivel del tracto gastrointestinal liberando el embrión hexacanto, el cual penetra la mucosa intestinal, siendo transportado a través de la sangre a diversos órganos donde se aloja, transformándose y desarrollándose posteriormente el estadio larvario (quiste hidatídico); los quistes son posteriormente ingeridos por el perro que se alimenta con vísceras contaminadas, desarrollando en aproximadamente 4045 días el gusano adulto a nivel intestinal ${ }^{(1-3)}$. En este ciclo de vida, los seres humanos cumplen la función de un hospedero intermediario accidental, desarrollando el estado larvario frecuentemente a nivel hepático (50 a $70 \%$ ), seguido por los pulmones (10 a $40 \%$ ) y otros lugares (de 15 a $20 \%)^{(1,2,4)}$.

Esta zoonosis se encuentra ampliamente distribuida en América del Sur (Perú, Argentina, Chile, sur de Brasil y Uruguay), las zonas que bordean el mar Mediterráneo, el sur y el centro de Rusia, Asia central, Australia y África ${ }^{(1,3)}$. La hidatidosis es considerada un serio problema de salud pública en estas regiones, sobre todo en áreas donde la principal actividad económica está en relación con la crianza de ganado, afectando la economía tanto de los individuos infectados y sus familias, así como de la sociedad en general ${ }^{(5,6)}$.

El riesgo de infección está altamente asociado con las interacciones entre humanos y perros, los cuales son usados principalmente como ayuda en el pastoreo del ganado; esta cercana interacción, así como determinadas creencias culturales y hábitos (matanza de animales en camales clandestinos, alimentar a los perros con vísceras crudas de animales infectados), contribuyen al mantenimiento de la transmisión del $E$. granulosus $^{(1,3,7-9)}$.

La sierra central y sur del Perú son zonas altamente endémicas para esta zoonosis; estudios realizados en población de áreas rurales de Junín, muestran prevalencias de hidatidosis alrededor de 5 a $9 \%$ usando ecografía abdominal y radiografía simple de tórax, y 10 a $18 \%$ usando serología por western blot ${ }^{(10-13)}$; existen varias hipótesis acerca de la gran proporción de seropositivos con resultado de imagen negativa (presencia de anticuerpos debido a una infección que no desarrollo lesión quística, o a un estadio temprano de enfermedad sin manifestación imagenológica al momento de la evaluación), por lo cual estudios realizados en campo sugieren el diagnóstico por imágenes como el mejor método de diagnóstico a ser usado en campo ${ }^{(14-16)}$.

En el Perú, a pesar de la alta prevalencia de hidatidosis reportada en diversos estudios de campo, no existe un programa nacional de control para la reducción de esta enfermedad. Para el desarrollo de un programa de control se requiere de información epidemiológica de base antes del establecimiento de las medidas de control (3); los estudios imagenológicos son caros y una definición preliminar de subgrupos de alto riesgo podría optimizar el uso de los recursos, mejorando la eficacia de los programas de despistaje necesarios para el establecimiento de una línea de base.

El objetivo principal del presente estudio es definir aquellas características del hogar que podrían ser consideradas como factores asociados al desarrollo de hidatidosis entre los miembros de un hogar. Estos factores pueden ser posteriormente usados en la definición de subgrupos de riesgo.

\section{MATERIALES Y MÉTODOS}

\section{ÁREA DE ESTUDIO}

Se realizó un estudio transversal en tres comunidades rurales de la región Junín (Chaquicocha, Ondores y Canchayllo) durante el año 2009 (Figura 1), como parte de una consultoría nacional para el establecimiento de un programa nacional de control; estas localidades se encuentran localizadas entre los 3200 y 3800 msnm y fueron seleccionadas por poseer la mayor población de

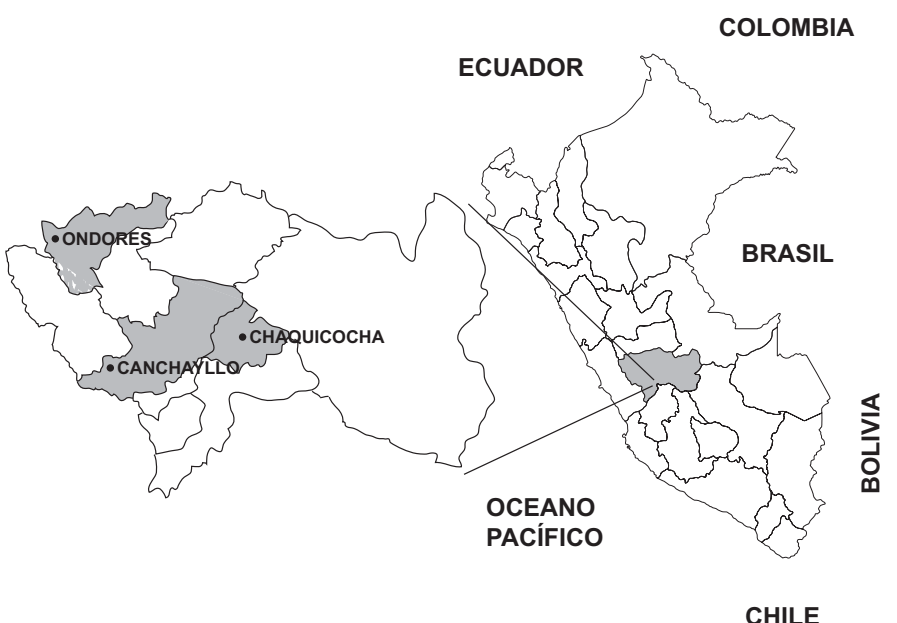

Figura 1. Ubicación de las comunidades de estudio: Ondores, Canchayllo y Chaquicocha localizadas en la región Junín, Perú. 


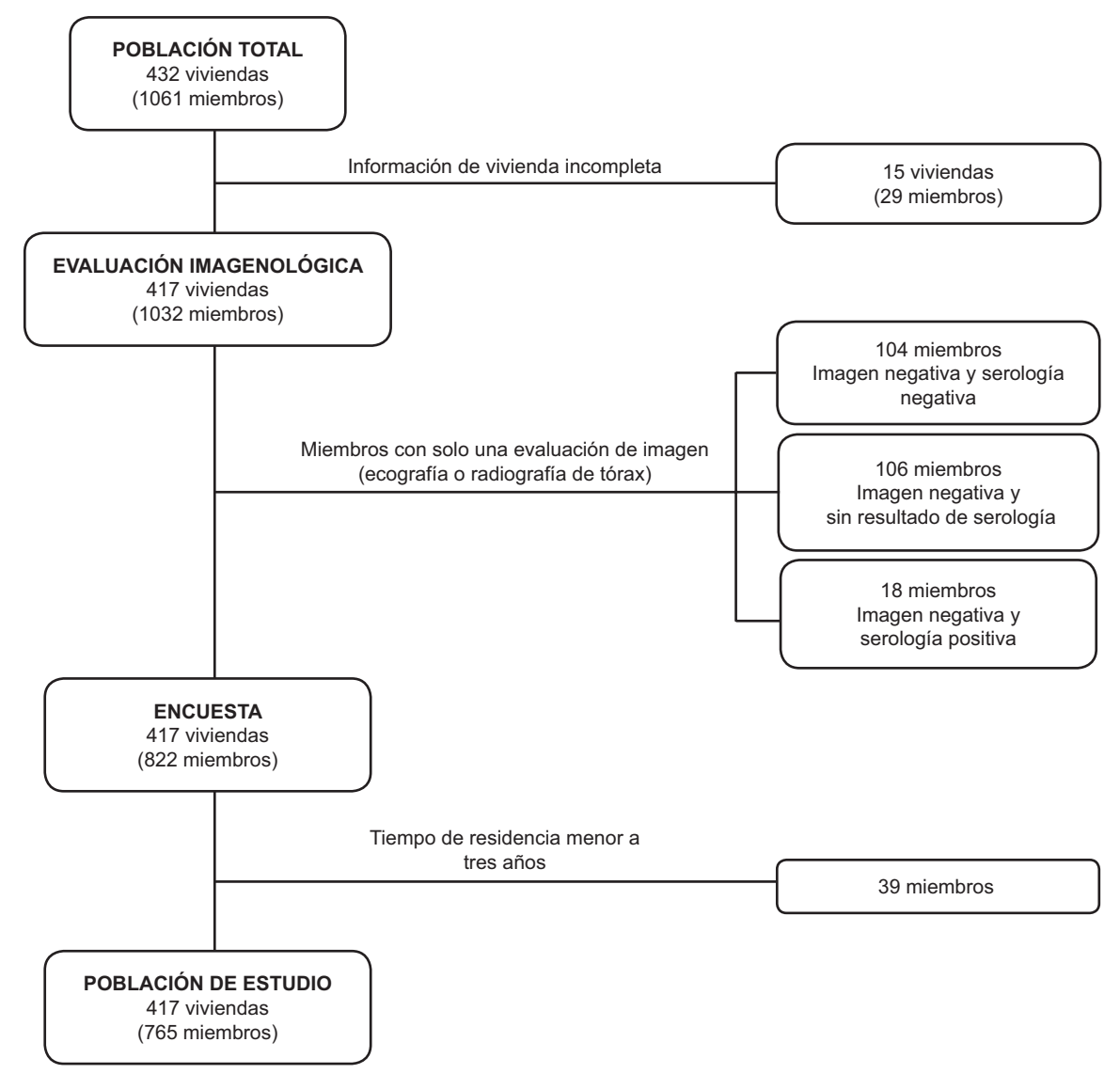

Figura 2. Selección de viviendas y miembros que participaron del estudio, Junín 2009.

ganado ovino en Junín, de acuerdo con ell último censo nacional agrario ${ }^{(17)}$.

\section{POBLACIÓN DE ESTUDIO}

En la selección de viviendas se tuvo en cuenta la existencia de información acerca de las características de la vivienda a ser evaluadas; por otro lado, en cada vivienda se seleccionó a aquellos miembros con un tiempo de residencia mayor a tres años y que de acuerdo con ell registro de cada casa, tengan ambos resultados de la evaluación imagenológica (ecografía abdominal y radiografía simple de tórax). Se excluyó del análisis aquellos hogares y miembros de la familia que no cumplían con los criterios arriba mencionados (Figura 2). La población final de estudio estuvo compuesta por 417 viviendas (765 miembros).

\section{PROCEDIMIENTOS}

La información fue recolectada mediante el uso de un cuestionario, el cual fue aplicado en los hogares al momento del censo e incluyó información acerca de las vivienda (número de habitantes, el tipo de suministro de agua, eliminación de excretas, crianza de animales y la presencia de perros), así como de sus miembros (edad de los miembros, sexo, número de años viviendo en la casa).

Adicionalmente a esta información, se solicitó el resultado de la evaluación imagenológica de cada uno de los miembros (positivo, negativo o indeterminado); dicho resultado fue definido por un médico radiólogo luego de observar los exámenes imagenológicos practicados a cada uno de los miembros.

\section{DEFINICIONES}

Se definió como positivo a la persona que presente al menos un resultado positivo para hidatidosis, independientemente del órgano afectado; como negativo a la persona que presente ambos resultados negativos; y como indeterminado a la persona que presente un solo resultado y este sea negativo, este último grupo no fue considerado para el análisis, dado que no cumplía con el criterio de inclusión referido anteriormente.

Con esta información se procedió a definir a las viviendas como positiva, aquella vivienda donde al menos un miembro sea positivo y como negativa, aquella vivienda 
donde todos los miembros sean negativos. Una variable adicional que se incluyó a las ya mencionadas fue la cobertura de evaluación, esta variable se definió como el porcentaje de miembros evaluados (miembros positivos + miembros negativos) del total de miembros registrados en la vivienda.

\section{ASPECTOS ÉTICOS}

El protocolo del estudio y los formularios para el consentimiento informado, información demográfica y epidemiológica, fueron aprobados por el Comité de Ética en Investigación del Instituto Nacional de Salud. Los datos fueron codificados para evitar la violación de la confidencialidad y la identificación de los participantes del estudio.

\section{ANÁLISIS DE DATOS}

Para las variables categóricas, se utilizó la prueba de Chi cuadrado o prueba exacta de Fisher según correspondía para comparar la frecuencia de las características del paciente; para las variables continuas se usó la prueba t de Student para comparar las medias. Se realizó un análisis de regresión logística simple (RLS), seguido de un análisis de regresión logística múltiple (RLM) para evaluar la asociación entre las características de las viviendas (número de habitantes, región donde se encuentra la casa, el abastecimiento de agua, tipo de eliminación de residuos, la crianza de ganado, tenencia de perro) y la probabilidad de tener al menos un caso positivo entre los miembros del hogar, esta asociación consideró la proporción de miembros evaluados (cobertura de evaluación) y las características de los participantes (edad media de los miembros evaluados, el sexo y el tiempo medio de residencia) como potenciales factores modificadores y confusores respectivamente. La colinearidad para el análisis de regresión logística se comprobó mediante la realización de un análisis de regresión múltiple en lugar de los análisis de regresión logística para calcular los factores de inflación de la varianza, los cuales eran todos muy por debajo de 5,0. Elegimos el mejor modelo basado en la prueba de razón de verosimilitud. Un valor de $p<0,05$ fue tomado para indicar significancia estadística. Todos los análisis se realizaron utilizando la versión de Stata v.10 (StataCorp LP College Station, TX, EE.UU.).

\section{RESULTADOS}

De las 417 viviendas evaluadas, 56 (13\%) tenían al menos un caso positivo entre sus miembros; de las 56 viviendas, cinco viviendas tenían dos miembros positivos, lo que hace un total de 61 (8\%) miembros positivos de 765 miembros evaluados. Las características de los hogares positivos y negativos se presentan en la Tabla 1. Más del $80 \%$ de los hogares contaban con menos de seis miembros, los hogares positivos tuvieron una media de edad de miembros evaluados y una media de tiempo de residencia mayor a lo observado en los hogares negativos $(44,9 \pm 23,1$ años frente a 36,4 $\pm 20,1$ años, y 39,8 $\pm 24,6$ años frente a $30,5 \pm 20,6$ años, respectivamente); además, se encontró una mayor proporción de hogares que refirieron crianza de animales y un mayor porcentaje de miembros evaluados a nivel de los hogares positivos en comparación con lo observado en los hogares negativos. Finalmente, la proporción de mujeres, eliminación de residuos, suministro de agua y contacto con perros, fue similar en ambos grupos.

El análisis de regresión logística simple (RLS) (Tabla 2) indicó asociación positiva, estadísticamente significativa, entre la probabilidad de tener al menos un caso positivo entre los miembros de un hogar y la ausencia de un suministro seguro de agua (OR = 2,61, IC 95\%: 1,14 $5,98 p,=0,023)$, crianza de animales (OR $=2,09$, IC 95\%: $1,04-4,18 p=0,038$ ), y comunidad donde el hogar estaba localizado. Los hogares ubicados en Ondores tuvieron una mayor asociación (OR = 2,49, IC 95\%: 1,19 - 5,21 $p=0,016)$. Por otro lado, se observó asociación entre la presencia de, al menos, un caso positivo en el hogar y el porcentaje de evaluación de los participantes por ambas técnicas imagenológicas (cobertura de evaluación). Finalmente, la edad media y el tiempo de residencia media de los miembros evaluados también mostraron una asociación positiva estadísticamente significativa.

No se encontró un efecto modificador de la variable "cobertura de la evaluación" con el resto de las covariables. Las variables que mostraron una asociación estadísticamente significativa en el análisis de RLS, fueron incluidas en el análisis de regresión logística múltiple (RLM), controlando este análisis por la variable "cobertura de evaluación" así como por las demás características del hogar (Tabla 2). Los resultados indicaron que los hogares con más de tres miembros localizados en Ondores y que reportaron crianza de animales fueron estadísticamente más propensos a tener al menos un caso positivo. Además, se observó una asociación negativa, estadísticamente significativa, entre la probabilidad de tener un caso positivo y el carecer de un sistema adecuado de eliminación de excretas (OR $=0,32,95 \%$ IC: $0,14-0,73, p=0,007)$.

\section{DISCUSIÓN}

El presente estudio analizó la asociación entre determinadas características de la vivienda y la probabilidad de 
Tabla 1. Características de los hogares evaluados, Junín, Perú 2009.

\begin{tabular}{|c|c|c|c|c|}
\hline & $\begin{array}{l}\text { Hogares positivos } \\
\qquad(n=56)\end{array}$ & $\begin{array}{l}\text { Hogares negativos } \\
\qquad(n=361)\end{array}$ & $\begin{array}{c}\text { Total } \\
(n=417)\end{array}$ & Valor $\mathbf{p}^{*}$ \\
\hline \multicolumn{5}{|l|}{ Número de habitantes - \% † } \\
\hline 1 a 3 & 42,9 & 39,1 & 39,6 & 0,846 \\
\hline 4 a 6 & 41,1 & 44,9 & 44,4 & \\
\hline$>6$ & 16,1 & 16,1 & 16,1 & \\
\hline \multicolumn{5}{|c|}{ Edad media de los miembros evaluados (años) } \\
\hline Media & 44,9 & 36,4 & 37,6 & 0,005 \\
\hline Desviación estándar & 23,1 & 20,1 & 20,7 & \\
\hline \multicolumn{5}{|l|}{ Suministro de agua- $\%$} \\
\hline Potable & 75,0 & 84,5 & 83,2 & 0,068 \\
\hline Pozo & 8,9 & 8,6 & 8,6 & \\
\hline Otro ${ }^{\ddagger}$ & 16,1 & 6,9 & 8,2 & \\
\hline \multicolumn{5}{|c|}{ Eliminación de excretas -\% } \\
\hline Baño & 35,7 & 24,1 & 25,7 & 0,192 \\
\hline Letrina & 32,2 & 36,3 & 35,7 & \\
\hline Otro $\S$ & 32,1 & 39,6 & 38,6 & \\
\hline \multicolumn{5}{|l|}{ Crianza de ganado-\% } \\
\hline $\mathrm{Si}$ & 80,6 & 66,2 & 68,1 & 0,044 \\
\hline \multicolumn{5}{|l|}{ Contacto con perros - $\%$} \\
\hline $\mathrm{Si}$ & 76,8 & 72,3 & 72,9 & 0,523 \\
\hline \multicolumn{5}{|l|}{ Cobertura de evaluación } \\
\hline $0 \%-25 \%$ & 12,5 & 30,8 & 28,3 & 0,018 \\
\hline $25 \%-50 \%$ & 37,5 & 34,6 & 35,0 & \\
\hline $50 \%-75 \%$ & 21,4 & 14,4 & 15,4 & \\
\hline $75 \%-100 \%$ & 28,6 & 20,2 & 21,3 & \\
\hline \multicolumn{5}{|c|}{ Tiempo medio de residencia (años) } \\
\hline Media & 39,8 & 30,5 & 31,7 & 0,002 \\
\hline Desviación estándar & 24,6 & 20,6 & 21,4 & \\
\hline \multicolumn{5}{|l|}{ Proporción de mujeres } \\
\hline$>0,5$ & 76,8 & 77,8 & 77,7 & 0,864 \\
\hline \multicolumn{5}{|l|}{ Comunidad } \\
\hline Ondores & 39,3 & 23,6 & 25,7 & 0,038 \\
\hline Canchayllo & 37,5 & 41,8 & 41,3 & \\
\hline Chaquicocha & 23,2 & 34,6 & 33,2 & \\
\hline
\end{tabular}

* Valores p, calculados usando el test exacto de Fisher, significancia $<0,05$.

† Número de miembros basado en el número total de miembros del hogar (evaluados y no evaluados).

$\mp$ Se considera otras fuentes de agua como río, acequia y manantial.

$\S$ Se considera otros lugares donde eliminar excretas; por ejemplo al aire libre.

tener al menos un caso positivo entre quienes la habitan. La población de estudio estuvo conformada por las viviendas de tres comunidades localizadas en la sierra central del Perú, zona altamente endémica para hidatidosis; se encontró una prevalencia, en viviendas e indi- vidual de 13 y $8 \%$ respectivamente; estudios previos que utilizaron métodos imagenológicos para el diagnóstico, y que fueron realizados en Junín, refieren prevalencias, en el ámbito individual, de lesiones quísticas parasitarias en hígado o pulmón de 5 a 9\% ${ }^{(10-12)}$. 
Tabla 2. Factores domiciliarios asociados con la presencia de hidatidosis humana en tres comunidades rurales de Junín, Perú.

\begin{tabular}{|c|c|c|c|c|c|c|}
\hline \multirow{2}{*}{ Variable } & \multicolumn{3}{|c|}{ Sin ajustar } & \multicolumn{3}{|c|}{ Modelo ajustado } \\
\hline & OR & IC $95 \%$ & $\mathbf{p}$ & OR & IC $95 \%$ & $\mathbf{p}$ \\
\hline \multicolumn{7}{|c|}{ Cobertura de la evaluación } \\
\hline $75 \%-100 \%$ & 3,48 & $1,36-8,86$ & 0,009 & 4,96 & $1,56-15,71$ & 0,006 \\
\hline $50 \%-75 \%$ & 3,66 & $1,36-9,84$ & 0,010 & 5,59 & $1,84-16,97$ & 0,002 \\
\hline $25 \%-50 \%$ & 2,66 & $1,09-6,51$ & 0,031 & 4,23 & $1,53-11,76$ & 0,006 \\
\hline $0-25 \%$ & 1 & & & 1 & & \\
\hline \multicolumn{7}{|l|}{ Número de personas * } \\
\hline$>6$ & 0,91 & $0,40-2,08$ & 0,826 & 2,54 & $0,87-7,38$ & 0,087 \\
\hline 4 a 6 & 0,83 & $0,45-1,54$ & 0,563 & 2,41 & $1,05-5,51$ & 0,037 \\
\hline 1 a 3 & 1 & & & 1 & & \\
\hline \multicolumn{7}{|l|}{ Comunidad } \\
\hline Ondores & 2,49 & $1,19-5,21$ & 0,016 & 2,36 & $1,05-5,31$ & 0,037 \\
\hline Canchayllo & 1,34 & $0,64-2,78$ & 0,436 & 0,82 & $0,31-2,14$ & 0,690 \\
\hline Chaquicocha & 1 & & & 1 & & \\
\hline Crianza de ganado & 2,09 & $1,04-4,18$ & 0,038 & 2,26 & $1,05-4,90$ & 0,038 \\
\hline \multicolumn{7}{|l|}{ Suministro de agua-\% } \\
\hline Otro $†$ & 2,61 & $1,14-5,98$ & 0,023 & 2,08 & $0,83-5,25$ & 0,117 \\
\hline Pozo & 1,17 & $0,43-3,17$ & 0,756 & 0,80 & $0,27-2,36$ & 0,69 \\
\hline Potable & 1 & & & 1 & & \\
\hline \multicolumn{7}{|c|}{ Eliminación de excretas } \\
\hline Otro $\ddagger$ & 0,55 & $0,27-1,09$ & 0,087 & 0,31 & $0,13-0,72$ & 0,007 \\
\hline Letrina & 0,60 & $0,30-1,19$ & 0,145 & 0,39 & $0,16-0,98$ & 0,046 \\
\hline Baño & 1 & & & 1 & & \\
\hline
\end{tabular}

* Número de miembros basado en el número total de miembros del hogar (evaluados y no evaluados).

† Se considera otras fuentes de agua como rio acequia, manantial.

$\ddagger$ Se considera otros lugares donde eliminar excretas; por ejemplo al aire libre.

Nuestro estudio se enfocó en evaluar las características del hogar como factores asociados con la presencia de enfermedad, para lo cual se realizó un análisis de RLS seguido de uno de RLM. El análisis de RLS mostró asociación de la mayoría de las características de la vivienda con la presencia de, al menos, un caso positivo en el hogar; las variables que mostraron asociación positiva fueron crianza de ganado, tiempo de residencia en la zona, edad promedio de los miembros del hogar, vivir en la comunidad con percentil de pobreza más bajo, la falta de un suministro adecuado de agua y una cobertura de evaluación imagenológica alta. Debido a que este análisis determina asociaciones que podrían deberse al efecto de otras covariables y no de la variable en sí (efecto confusor o efecto modificador) se controló las asociaciones encontradas mediante un análisis de RLM, el cual mostró que las variables número de personas en el hogar, crianza de ganado y vivir en una comunidad pobre, mantuvieron la asociación positiva encontrada en el análisis de RLS, lo cual no fue el caso del tiempo de residencia media, edad media de los miembros del hogar y suministro seguro de agua. Adicionalmente, tras del análisis de RLM, se observó una asociación negativa, estadísticamente significativa, entre la carencia de un adecuado sistema de eliminación de excretas $(O R=0,31)$ y la presencia de al menos un caso positivo en el hogar.

En relación con las características que presentaron asociación positiva luego del análisis de RLM, la única característica que ha sido descrita como factor de riesgo en estudios previos, fue la crianza de animales $(3,8,18)$. Las otras dos características (ausencia de un suministro seguro de agua y vivir en la comunidad con quintil de pobreza más bajo) no han sido descritas como tales en estudios previos, pero estarían directamente relacionadas con la pobreza, lo cual sí ha sido discutido en otros estudios donde se reportó que algunas características socioeconómicas y culturales serían los mejores factores asociados con la presencia de hidatidosis, nombrándose como ejemplo el contacto cercano con perros sin control veterinario, el sacrificio del ganado sin control y las condiciones insalubres de vida ${ }^{(19-21)}$. Esta asociación entre la pobreza y el desarrollo de la enfermedad ha ido ganando la atención en los últimos años y, hoy en día, el alivio de la pobreza es uno de los objetivos propuestos por la OMS-FAO para el control de las zoonosis ${ }^{(22)}$. Finalmente, tras el análisis de 
RLM, se encontró asociación negativa entre la carencia de un sistema adecuado de eliminación de excretas y la presencia de al menos un caso positivo en la vivienda, esta variable no ha sido reportada en estudios previos como factor protector; sin embargo, en nuestro caso la asociación negativa encontrada se podría deber a un problema en la recolección de la variable por parte de los entrevistadores, dado que en comunidades rurales se suele confundir letrina con pozo ciego y con presencia de inodoro pero sin un sistema de alcantarillado, y además, en estas zonas el uso de letrinas públicas podrían ser consideradas como eliminación de excretas en el campo, por lo que se recomienda su exploración de manera más adecuada en algún estudio posterior.

Por otro lado, los factores de riesgo asociados con la enfermedad como son el contacto con perros, el sexo y la edad, que han sido descritos en otras publicaciones $(3,8,23-25)$, no mostraron asociación positiva en nuestro estudio. En el caso del contacto con perros, el que no se encontrara asociación positiva se puede deber a que la asociación se da más de modo individual, lo cual ha sido descrito en estudios donde se evalúan los conocimientos actitudes y prácticas de manera individual en relación al contacto con el perro, determinando que la probabilidad de enfermedad varía con relación al grado de interacción entre el perro y el individuo, ya sea por la ocupación de las personas, los hábitos higiénico alimenticios, la práctica de matanza de ganado en el hogar, la inadecuada eliminación de vísceras contaminadas y el cuidado y alimentación de los perros con vísceras contaminadas $^{(8,26)}$. Con relación al sexo, estudios previos han documentado que ser mujer mayor a 40 años de edad presenta una asociación positiva con desarrollar hidatidosis (27); esto probablemente está relacionado con las actividades que desarrolla la mujer dentro del hogar, así como una mayor exposición a perros infectados dentro del hogar. Otra de las variables descritas como factor de riesgo ha sido la edad, describiéndose en algunos casos un mayor riesgo a mayor edad, sin embargo, esta variable tendría un comportamiento individual y lo encontrado en nuestro caso en el análisis de RLS se explicaría por el efecto confusor de las covariables tiempo y lugar de residencia, dado que a mayor edad, mayor tiempo de residencia en un área endémica, lo que incrementa el tiempo de exposición y también el riesgo; además, a esto se agregaría que a mayor edad, mayor tiempo para que la enfermedad se manifieste clínica o imagenológicamente, ya que los quistes hidatídicos crecen muy lentamente y suelen tener un tiempo de latencia largo ${ }^{(1,2,4)}$.

Se realizó un estudio transversal, por lo tanto, su principal limitación es que las asociaciones encontradas no pueden definirse como factores de riesgo, dado que para esto se debería evaluar el factor de temporalidad, el cual no ha sido evaluado en nuestro estudio. Por otro lado, se solicitó la lista de participantes positivos de acuerdo con el criterio antes mencionado, criterio que se basó en la información publicada tanto a nivel local como internacional, la cual refiere que en estudios de campo el mejor criterio para definir un caso debe basarse en el resultado de la evaluación imagenológica, dada su alta sensibilidad (88-98\%) y especificidad (93-100\%) en comparación con la evaluación serológica $(12,14,16,28)$; el problema con la selección de miembros radica en la exclusión de un gran número de participantes ( $n=267$ ), dentro de los cuales se excluyó a 18 participantes que tuvieron resultado serológico positivo, sin imagen que descarte o confirme el diagnóstico serológico. La exclusión de estos miembros afectaría el grado de asociación observado en nuestro análisis; sin embargo, el efecto no sería grande si se consideran que los casos excluidos mantienen la misma proporción de positivos que los casos incluidos en el análisis ( $8 \%)$, para tal efecto se asumiría que los seropositivos $(n=18)$ serían positivos y los restantes $(n=249)$ serían negativos en ambas evaluaciones imagenológicas, teniendo una proporción de positivos (prevalencia) de $\sim 7 \%$, valor que, además, se encuentra dentro del rango descrito para la zona (5 a $9 \%$ ). No obstante, debe ser tomado con cautela y debería ser considerado en la interpretación de los resultados.

En conclusión, luego del análisis realizado, se puede observar que los hogares con más de tres miembros localizados en Ondores (comunidad ubicada en el quintil más bajo de pobreza), con una falta de adecuado suministro de agua, que refieren crianza de animales tienen asociación positiva de tener un caso positivo entre sus miembros en comparación con otros hogares. Estas características deben ser consideradas en una evaluación posterior con la finalidad de evaluar su potencial papel como factores de riesgo, ayudando de esta manera a la determinación de grupos de alto riesgo, lo cual en una actividad de despistaje, ya sea para el establecimiento de una línea de base o para el seguimiento de una población bajo un programa de control, seria de mucha utilidad en términos del uso apropiado de los recursos.

\section{Financiamiento}

Este trabajo fue desarrollado por el consorcio formado por la Facultad de Medicina Veterinaria de la Universidad Nacional Mayor de San Marcos, la Universidad Peruana Cayetano Heredia y el Instituto Peruano de Parasitología Clínica y Experimental - INPPACE con el financiamiento del Instituto Nacional Salud de Perú dentro del marco del Programa de Investigación de Hidatidosis en Zonas Endémicas. 


\section{Conflictos de Interés}

Los autores declaran no tener conflictos de interés en la publicación de este artículo.

\section{REFERENCIAS BIBLIOGRÁFICAS}

1. Moro P, Schantz PM. Echinococcosis: a review. Int J Infect Dis. 2009;13(2):125-33.

2. Brunetti E, Junghanss T. Update on cystic hydatid disease. Curr Opin Infect Dis. 2009;22(5):497-502.

3. Craig PS, McManus DP, Lightowlers MW, Chabalgoity JA, Garcia HH, Gavidia CM, et al. Prevention and control of cystic echinococcosis. Lancet Infect Dis. 2007;7(6):385-94.

4. Brunetti E, Kern P, Vuitton DA. Expert consensus for the diagnosis and treatment of cystic and alveolar echinococcosis in humans. Acta Trop. 2009;114(1):1-16.

5. Torgerson PR. Economic effects of echinococcosis. Acta Trop. 2003;85(2):113-18.

6. Budke CM, Jiamin Q, Qian W, Torgerson PR. Economic effects of echinococcosis in a disease-endemic region of the Tibetan Plateau.. Am J Trop Med Hyg. 2005;73(1):2-10.

7. Wang Z, Wang $\mathbf{X}$, Liu X. Echinococcosis in China, a review of the epidemiology of Echinococcus spp. Ecohealth. 2008;5(2):115-26.

8. Moro PL, Cavero CA, Tambini M, Briceno Y, Jimenez R, Cabrera L. Identification of risk factors for cystic echinococcosis in a peri-urban population of Peru. Trans $\mathrm{R}$ Soc Trop Med Hyg. 2008;102(1):75-8.

9. Yang YR, Sun T, Li Z, Zhang J, Teng J, Liu X, et al. Community surveys and risk factor analysis of human alveolar and cystic echinococcosis in Ningxia Hui Autonomous Region, China. Bull World Health Organ. 2006;84(9):714-21.

10. Moro PL, McDonald J, Gilman RH, Silva B, Verastegui M, Malqui V, et al. Epidemiology of Echinococcus granulosus infection in the central Peruvian Andes. Bull World Health Organ. 1997;75(6):553-61.

11. Moro PL, Guevara A, Verastegui M, Gilman RH, Poma H, Tapia B, et al. Distribution of hydatidosis and cysticercosis in different Peruvian populations as demonstrated by an enzyme-linked immunoelectrotransfer blot (EITB) assay. The Cysticercosis Working Group in Peru (CWG). Am J Trop Med Hyg. 1994;51(6):851-55.

12. Moro PL, Garcia HH, Gonzales AE, Bonilla JJ, Verastegui M, Gilman RH. Screening for cystic echinococcosis in an endemic region of Peru using portable ultrasonography and the enzyme-linked immunoelectrotransfer blot (EITB) assay. Parasitol Res. 2005;96(4):242-46.

13. Moro PL, Bonifacio N, Gilman RH, Lopera L, Silva B, Takumoto $\mathbf{R}$, et al. Field diagnosis of Echinococcus granulosus infection among intermediate and definitive hosts in an endemic focus of human cystic echinococcosis. Trans R Soc Trop Med Hyg. 1999;93(6):611-15.

14. Macpherson $\mathbf{C N}$, Milner R. Performance characteristics and quality control of community based ultrasound surveys for cystic and alveolar echinococcosis. Acta Trop. 2003;85(2):203-9.
15. Macpherson CN, Bartholomot B, Frider B. Application of ultrasound in diagnosis, treatment, epidemiology, public health and control of Echinococcus granulosus and $E$. multilocularis. Parasitology. 2003;127(Suppl): S21-35.

16. Gavidia CM, Gonzalez AE, Zhang W, McManus DP, Lopera L, Ninaquispe B, et al. Diagnosis of cystic echinococcosis, central Peruvian Highlands. Emerging infectious diseases. 2008;14(2):260-66.

17. Instituto Nacional de Estadística e Informática. III Censo Nacional Agropecuario 1994 (CENAGRO). Resultados Censales 1994 Lima: INEI; 1994.

18. García-Apaico V, Vargas-Cuba FH, Martínez-Salcedo J, Huamani-Basilio N, Fernández-Chillcce I, LaraRomaní E. Seroprevalencia de Hidatidosis en Escolares de Huancasancos, Ayacucho 2004. Rev Peru Med Exp Salud Publica. 2008;25(3):290-3.

19. Schantz PM, Wang H, Qiu J, Liu FJ, Saito E, Emshoff A, et al. Echinococcosis on the Tibetan Plateau: prevalence and risk factors for cystic and alveolar echinococcosis in Tibetan populations in Qinghai Province, China. Parasitology. 2003;127(Suppl): S109-20.

20. Carmona C, Perdomo R, Carbo A, Alvarez C, Monti J, Grauert R, et al. Risk factors associated with human cystic echinococcosis in Florida, Uruguay: results of a mass screening study using ultrasound and serology. Am J Trop Med Hyg. 1998;58(5):599-605.

21. Burridge MJ, Schwabe CW, Fraser J. Hydatid disease in New Zealand: changing patterns in human infection, 18781972. N Z Med J. 1977;85(583):173-77.

22. Robinson A. Veterinary public health and control of zoonoses in developing world. Roma: FAO; 2003

23. Campos-Bueno A, Lopez-Abente G, Andres-Cercadillo AM. Risk factors for Echinococcus granulosus infection: a case-control study. Am J Trop Med Hyg. 2000;62(3):329-34.

24. Núñez E, Calero D, Estares L, Morales A. Prevalencia y factores de riesgo de hidatidosis en población general del distrito de Ninacaca-Pasco. An Fac Med (Lima). 2003;64(1):34-42.

25. Larrieu EJ, Costa MT, del Carpio M, Moguillansky S, Bianchi G, Yadon ZE. A case-control study of the risk factors for cystic echinococcosis among the children of Rio Negro province, Argentina. Ann Trop Med Parasitol. 2002;96(1):43-52.

26. Nasrieh MA, Abdel-Hafez SK, Kamhawi SA, Craig PS, Schantz PM. Cystic echinococcosis in Jordan: socioeconomic evaluation and risk factors. Parasitology Res. 2003;90(6):456-66.

27. Tiaoying L, Jiamin Q, Wen Y, Craig PS, Xingwang C, Ning $\mathbf{X}$, et al. Echinococcosis in Tibetan populations, western Sichuan Province, China. Emerg Infect Dis. 2005;11(12):1866-73.

28. Torgerson PR, Deplazes P. Echinococcosis: diagnosis and diagnostic interpretation in population studies. Trends Parasitol. 2009; 25(4):164-70.

Correspondencia: Dr. Saul J. Santivañez.

Dirección: Av Primavera 120, oficina A-210. Chacarilla -

Santiago de Surco, Lima 33, Perú.

Correo electrónico: ssantiva@jhsph.edu 